\title{
ARTICLE \\ Donepezil, a drug for Alzheimer's disease, promotes oligodendrocyte generation and remyelination
}

\author{
Xue Cui ${ }^{1}$, Yu-e Guo ${ }^{2,3}$, Jia-hui Fang ${ }^{1}$, Chang-jie Shi ${ }^{1}$, Na Suo ${ }^{2,3}$, Ru Zhang ${ }^{1}$ and Xin Xie ${ }^{1,2,4}$
}

\begin{abstract}
Myelin sheaths play important roles in neuronal functions. In the central nervous system (CNS), the myelin is formed by oligodendrocytes (OLs), which are differentiated from oligodendrocyte precursor cells (OPCs). In CNS demyelinating disorders such as multiple sclerosis (MS), the myelin sheaths are damaged and the remyelination process is hindered. Small molecule drugs that promote OPC to OL differentiation and remyelination may provide a new way to treat these demyelinating diseases. Here we report that donepezil, an acetylcholinesterase inhibitor (AChEl) developed for the treatment of Alzheimer's disease (AD), significantly promotes OPC to OL differentiation. Interestingly, other AChEls, including huperzine $A$, rivastigmine, and tacrine, have no such effect, indicating that donepezil's effect in promoting OPC differentiation is not dependent on the inhibition of AChE. Donepezil also facilitates the formation of myelin sheaths in OPC-DRG neuron co-culture. More interestingly, donepezil also promotes the repair of the myelin sheaths in vivo and provides significant therapeutic effect in a cuprizone-mediated demyelination animal model. Donepezil is a drug that has been used to treat AD safely for many years; our findings suggest that it might be repurposed to treat CNS demyelinating diseases such as MS by promoting OPC to OL differentiation and remyelination.
\end{abstract}

Keywords: donepezil; aricept; oligodendrocyte; oligodendrocyte progenitor cell; differentiation; myelin sheaths; remyelination; CNS demyelinating disorders; multiple sclerosis

Acta Pharmacologica Sinica (2019) 40:1386-1393; https://doi.org/10.1038/s41401-018-0206-4

\section{INTRODUCTION}

Oligodendrocytes (OLs), an important component of neuroglia, form myelin sheaths that exert trophic and metabolic support to encased axons in the central nervous system (CNS) [1, 2]. Myelinating OLs are generated from oligodendrocyte precursor cells (OPCs) [3], which are characterized by the expression of proteoglycan glial antigen 2 (NG2) and platelet derived growth factor receptor alpha (PDGFRa). In contrast to most precursor cells, OPCs are situated abundantly throughout the adult CNS and retain the ability to generate new OLs to reform myelin that might be lost with age or disease $[4,5]$. In some neurodegenerative diseases, such as multiple sclerosis, demyelination and axonal damage disrupt the saltatory nerve conduction; moreover, the failure to reform the myelin eventually leads to neurological disability [6]. Extensive studies to date suggest that the failure of remyelination is likely due to the loss of OLs in the lesions and the failure of OL generation from OPCs [7-9]. It is therefore imperative to develop treatments that target facilitating remyelination.

A number of small molecule compounds have been reported to promote OPC to OL differentiation. Benztropine, an anticholinergic drug used for the treatment of Parkinson's disease (PD), has recently been demonstrated to promote OPC to OL differentiation and remyelination through direct antagonism of $\mathrm{M} 1$ and/or $\mathrm{M} 3$ muscarinic receptors [10]. Olesoxime, a compound that regulates mitochondria function, has been found to accelerate OL maturation in culture and increase remyelination in rodents [11, 12]. Our previous studies also discovered that U50488, a kappa opioid receptor agonist, and Vitamin C, promote OPC to OL differentiation in vitro and remyelination in vivo $[13,14]$. Using a similar screening strategy $[13,14]$, we also identified that donepezil, a drug used for Alzheimer's disease (AD), significantly enhances OPC to OL differentiation.

Donepezil is an acetylcholinesterase inhibitor (AChEI). Similar to donepezil, several AChEls, including huperzine A, rivastigmine, tacrine, physostigmine, galantamine, and ganstigmine, are approved to treat moderate $A D$ by inhibiting the breakdown of acetylcholine, a neurotransmitter associated with memory and widely distributed in the CNS $[15,16]$. Apart from blocking AChE, donepezil has been reported to have other biological functions. Donepezil may protect neurons via direct and indirect stimulation of nicotinic acetylcholine receptors (nAChRs) against glutamateinduced neurotoxicity $[17,18]$. Direct binding to Sigma-1 receptor might be involved in donepezil's rescue effect on $A \beta$-caused hippocampal long-term potentiation impairment [19]. Moreover, it has been confirmed that donepezil alleviates the Lipopolysaccharides (LPS)-induced inflammatory response and suppresses nuclear translocation of nuclear factor-kappa $\mathrm{B}(\mathrm{NF}-\mathrm{kB})$ in the murine macrophage cell line RAW 264.7 [20]. However, whether donepezil

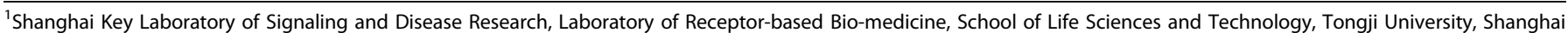

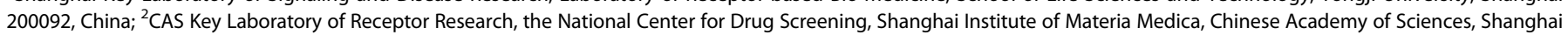

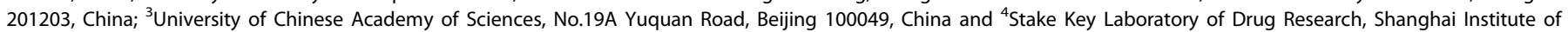
Materia Medica, Chinese Academy of Sciences, Shanghai 201203, China

Correspondence: Ru Zhang (ru.zhang@tongji.edu.cn) or Xin Xie (xxie@simm.ac.cn)
}

Received: 29 June 2018 Accepted: 19 December 2018

Published online: 27 March 2019 
or other AChEls could affect OPC to OL differentiation or myelination remains unclear.

We report that donepezil, but not other AChEls, significantly promotes OPC to OL differentiation. Donepezil also facilitates the formation of myelin sheaths in OPC-DRG (dorsal root ganglion) neuron co-culture. More interestingly, donepezil promotes the repair of myelin sheaths in vivo and provides a significant therapeutic effect in a cuprizone-mediated demyelination animal model.

\section{MATERIALS AND METHODS}

Chemicals and reagents

Tacrine (9-amino-1,2,3,4-tetrahydroacridine hydrochloride hydrate) and rivastigmine $L$-tartrate $\left(\mathrm{C}_{14} \mathrm{H}_{22} \mathrm{~N}_{2} \mathrm{O}_{2} \cdot \mathrm{C}_{4} \mathrm{H}_{6} \mathrm{O}_{6}\right)$ were generously supplied by Haiyan Zhang research group of Shanghai Institute of Materia Medica, Chinese Academy of Sciences. Donepezil hydrochloride monohydrate, paraformaldehyde (PFA), bis(cyclohexanone)oxaldihydrazone (Cuprizone), Hoechst $33342, n$-acetylcysteine, poly- $D L$-ornithine hydrobromide, laminin, papain, $L$-cysteine, insulin, transferrin, progesterone, putrescine, BSA, 5-fluoro-2'-deoxyuridine, and triiodothyronine (T3) were purchased from Sigma-Aldrich (St. Louis, MO, USA). (-)-Huperzine A was purchased from Selleck (Houston, USA). Rat tail collagen, collagenase II, and dispase II were purchased from Roche (Basel, Switzerland). Human PDGF-AA, human fibroblast growth factorbasic (FGF2), and recombinant human EGF were obtained from PeproTech EC (London, UK).

OPC differentiation from NPCs

All animal experiments were performed in accordance with protocols approved by the Animal Care and Use Committee of Tongji University. Neural progenitor cell (NPC)-derived OPCs were generated by culture of cortical NPCs with OPC induction conditions. Briefly, the cortices were removed from E14.5 embryonic mouse brain and mechanically dissociated into single cells. The cells were then expanded in NPC medium (DMEM/F12 media (Gibco), 2\% B27, $20 \mathrm{ng} / \mathrm{mL}$ EGF, and $20 \mathrm{ng} / \mathrm{mL}$ FGF2) for 2 days until neurosphere formation. The neurospheres were passaged every 2 days. All experiments were performed using passage 3 (P3) NPCs. The neurospheres were dispersed into singlecell suspensions by accutase (Millipore, SF006) digestion and were split into OPC medium (DMEM/F12 media, 2\% B27, $10 \mathrm{ng} / \mathrm{mL}$ FGF2, and $20 \mathrm{ng} / \mathrm{mL}$ PDGF-AA) for 2 days to produce OPCs from NPCs.

\section{High-throughput screening}

For the primary screening, the single-cell suspension of NPCs was seeded at a density of 8000 cells per well onto 96 -well plates coated by poly-ornithine $(5 \mu \mathrm{g} / \mathrm{mL})$ and laminin $(1 \mu \mathrm{g} / \mathrm{mL})$. OPC medium was used for 2 days, and the medium was subsequently changed to basal medium (DMEM/F12 supplemented with $2 \%$ B27) that contained various compounds with a final concentration of $20 \mu \mathrm{M}$ for an additional 4.5 days. Thyroid-hormone T3 (100 nM) and DMSO $(0.2 \%, v / v)$ were used as the positive and vehicle controls, respectively, in each plate. The cells were then fixed with 4\% PFA at room temperature and incubated with an anti-MBP antibody (Abcam, ab7349) and a secondary antibody conjugated to Alexa Fluor 488 (Thermo Fisher, A-11001). The nuclei were stained with Hoechst 33342. Eleven images per well (representing different locations in a single well) were captured, and the nuclei and myelin basic protein (MBP)-positive cells were quantified using the Operetta high content analysis system (PerkinElmer).

Immunofluorescence staining

Cells were fixed with 4\% PFA in phosphate buffered saline (PBS) for $15 \mathrm{~min}$ at room temperature. After being washed three times with PBS, the cells were permeabilized and blocked with PBS that contained $1 \%$ BSA and $0.1 \%$ Triton (for 04 staining, Triton was omitted) for $30 \mathrm{~min}$ at room temperature. The cells were subsequently incubated with the corresponding primary antibodies at $4{ }^{\circ} \mathrm{C}$ overnight. After washing, the cells were incubated with the appropriate fluorescence-conjugated secondary antibodies for $1 \mathrm{~h}$ at room temperature. Hoechst 33342 was used to identify cell nuclei. The antibodies used in this assay are as follows: anti-Nestin (Millipore, MAB353, 1:400), anti-PDGFRa (Cell signaling, 3164S, 1:200), anti-Olig2 (Millipore, AB9610, 1:200), anti-NG2 (Millipore, AB5320, 1:200), anti-MBP (Abcam, ab7349, 1:500), and anti-NF-200 (Sigma, N4142 and N5389, 1:1000).

OPC-DRG neuron co-culture

DRG neurons isolated from postnatal (P5-P10) mice were incubated in Hank's balanced salt solution (HBSS) that contained papain $(3 \mathrm{U} / \mathrm{mL})$ and $L$-cysteine $(0.36 \mathrm{mg} / \mathrm{mL})$ for $10 \mathrm{~min}$ at $37^{\circ} \mathrm{C}$. After removal of the papain solution, the DRG neurons were further incubated in HBSS that contained collagenase II $(100 \mathrm{U} / \mathrm{mL})$ and dispase $\|(2 \mathrm{U} / \mathrm{mL})$ for $10 \mathrm{~min}$ at $37^{\circ} \mathrm{C}$. After thorough washing, the dissociated DRG neurons were seeded at a density of 20000 cells per well onto poly-D-lysine $(500 \mu \mathrm{g} / \mathrm{mL})$ and rat tail collagen $(25 \mu \mathrm{g} / \mathrm{mL})$ coated 48 -well plates and maintained in OLmedium (DMEM, 2\% B27, glutamax, $5 \mu \mathrm{g} / \mathrm{mL}$ insulin, $50 \mu \mathrm{g} / \mathrm{mL}$ transferrin, $0.5 \% \mathrm{FBS}, 0.2 \mu \mathrm{M}$ progesterone, $100 \mu \mathrm{M}$ putrescine, 0.1 $\mathrm{mg} / \mathrm{mL}$ BSA, $100 \mathrm{U} / \mathrm{mL}$ penicillin, and $100 \mu \mathrm{g} / \mathrm{mL}$ streptomycin) for 9 days. 5-Fluoro-2'-deoxyuridine $(10 \mu \mathrm{M})$ was added to remove contaminating glia cells for the first 7 days. After 9 days, 30000 OPCs isolated from neonatal mouse cortices with anti-AN2 microbeads (Miltenyi) were added per well to DRG neurons, and the co-cultures were maintained for an additional 9 days in $\mathrm{OL}$ medium that contained indicated drugs. The cultures were fixed and stained with anti-NF-200 (Sigma, N4142), anti-MBP (Abcam, ab7349). For cell imaging, ten pictures per well were obtained and analyzed by the Operetta high content analysis system. Myelination was identified as neurites double positive for MBP and NF-200 staining.

\section{Reverse transcription and PCR}

Total RNA was extracted with TRIzol (Invitrogen) and was subjected to reverse transcription with random hexamer primer and Moloney murine leukemia virus reverse transcriptase (Promega). Realtime PCR was conducted in a LightCycler quantitative PCR apparatus (Stratagene) using the FastStart Universal SYBR Green Master (Rox). All gene expression results are expressed as arbitrary units relative to $\beta$-actin expression. The primer sequences used were as follows: PLP, forward $5^{\prime}$-TGCTCGGCTGTACCTGTGTA CATT-3' and reverse $5^{\prime}$-TACATTCTGGCA TCAGCGCAGAGA-3'; MBP, forward 5'-TGACACCTCGAACACCACCTC- $3^{\prime}$ and reverse $5^{\prime}$-CCT TGAA TCCCTTGTGAGCC-3'; MAG, forward 5'-GGTGTTGAGGGAGG CAGTTG- $3^{\prime}$ and reverse $5^{\prime}$-CCTTGAATCCCTTGTGAGCC-3'; and CNPase, forward 5'-TCCACGAGTGCAAGACGCTATTCA-3' $3^{\prime}$ and reverse 5'-TGTAAGCATCAGCGGACACCATCT-3'.

\section{Western blot}

NPC-derived OPCs and OLs were collected in lysis buffer $(50 \mathrm{mM}$ Tris- $\mathrm{HCl}, 2 \% \mathrm{w} / \mathrm{v}$ SDS, $10 \%$ glycerol, $1 \% \beta$-mercaptoethanol, and $0.01 \%$ bromophenyl blue $(\mathrm{pH} 6.8)$ ) and boiled at $95-100^{\circ} \mathrm{C}$ for 10 min. The samples were separated on SDS-PAGE and transferred onto $0.2 \mu \mathrm{m}$ polyvinylidene difluoride (PVDF, Millipore) membranes. The membranes were first incubated in blocking buffer (TBS with $0.05 \%$ Tween 20 and $5 \%$ nonfat milk) for $1 \mathrm{~h}$ at room temperature and then incubated with a rabbit anti-GAPDH (CST, $1: 10,000$ ) or a rat anti-MBP (Abcam, ab7349, 1:1000) antibody overnight at $4{ }^{\circ} \mathrm{C}$. After thorough washing, the membranes were incubated with the appropriate HRP-conjugated secondary antibodies for $1 \mathrm{~h}$ at room temperature. After washing, immunoblotting signals were visualized using Amersham ECL Plus Western Blotting detection reagents (GE Healthcare). 
Cuprizone-induced demyelination mouse model

Female C57BL/6 mice (8 weeks) maintained in pathogen-free conditions were fed with $0.2 \%(\mathrm{w} / \mathrm{w})$ cuprizone (Sigma) mixed with ground standard rodent chow. The cuprizone diet was maintained for 5 weeks, following which the cuprizone-infused feed was removed and the animals were given standard chow. The mice were administered a daily intraperitoneal injection of donepezil (1.5 and $5 \mathrm{mg} / \mathrm{kg}$ ) after cuprizone withdrawal. The treatment lasted for 1-3 weeks. The animals were subsequently anesthetized with $5 \%$ chloral hydrate and sacrificed. The brains were carefully separated, paraffin-embedded, sectioned, and stained for histopathological analysis.

Histology and immunofluorescent analysis

Paraffin-embedded coronal sections $(5 \mu \mathrm{m})$ of the brains were stained with Luxol fast blue (LFB, Sigma, S3382) for the analysis of demyelination. Images were obtained, and quantitative image analysis was performed using ImagePro software. The region of interest corresponding to the corpus callosum was initially drawn using the "irregular AOl" tool, and non-blue areas were then counted within the lesion using the "count and measure objects" tool. The percent of the demyelination area was calculated by the ratio of the non-blue area to the total corpus callosum area. For immunofluorescent analysis, paraffin-embedded coronal sections of the brains were permeabilized and blocked with PBS that contained $2 \% \quad \mathrm{BSA}$ and $0.3 \%$ Triton for $30 \mathrm{~min}$ at room temperature, followed by incubation with a mouse anti-MBP antibody (Abcam, ab7349, 1:500), a rabbit polyclonal anti-GST-pi antibody (Millipore, AB5320, 1:200), or a rabbit anti-Olig2 antibody (Millipore, $A B 9610,1: 200)$ at $4{ }^{\circ} \mathrm{C}$ overnight. After thorough washing, the sections were stained with secondary antibodies conjugated to Alexa Fluor 488 (Thermo Fisher, 1:1,000), and the nuclei were stained with Hoechst 33342. Images were obtained using an Olympus IX71 inverted fluorescent microscope, and quantitative image analysis was performed using ImagePro software.

\section{Electron microscopy}

Brains were isolated from 4\% PFA perfused mice; the corpus callosum was isolated and fixed in PBS that contained $2.5 \%$ glutaraldehyde for $2 \mathrm{~h}$. The corpus callosum was subsequently washed, fixed in 1\% osmium tetroxide, dehydrated in acetone, and embedded in EPON. Then, 70-nm thin sagittal sections were cut with a diamond knife and mounted on copper slot grids coated with Formvar and stained with uranyl acetate and lead citrate for examination on a JEM-1230 transmission electron microscope. The G-ratios were analyzed in ImagePro, and approximately 200 remyelinated axons were assessed for each group.

\section{Statistical analysis}

Data were analyzed with GraphPad Prism software. For comparison between two groups, statistical evaluation was performed by two-tailed Student's $t$-test or two-way analysis of variance was used to assess the significance between treatment groups of the cuprizone-induced mouse model of demyelination. For all statistical tests, $P$ values $<0.05$ were considered statistically significant. All error bars show the standard error of the mean (SEM).

\section{RESULTS}

Generation of OLs from NPC-derived OPCs

We established a simple protocol to induce OL differentiation from NPC-derived OPCs (Fig. 1a). Cortical neurospheres that expressed nestin were generated from mouse E14.5 embryos, which could be propagated and passaged in NPC medium that contained bFGF and EGF (Fig. 1b). The NPCs were then cultured in
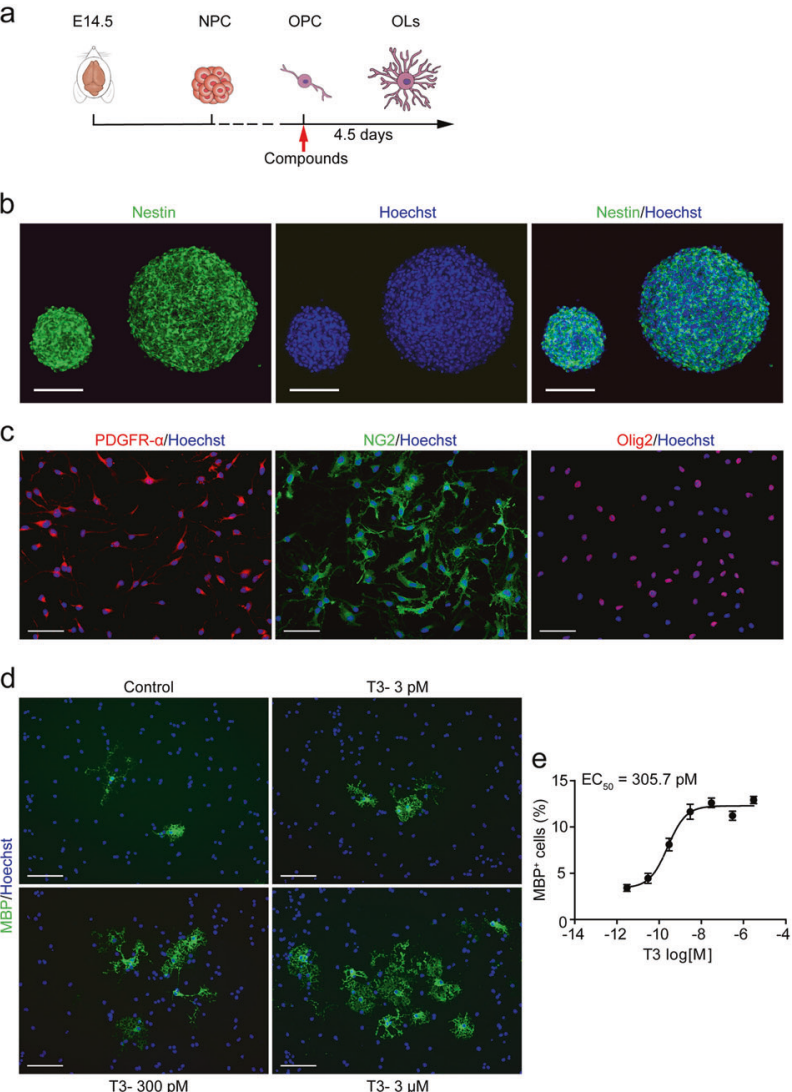

Fig. 1 NPC-based differentiation assay to identify modulators of OL differentiation and maturation. a Protocol for the generation of OLs from cortical NPCs isolated from mouse E14.5 embryos. b Immunofluorescent staining of the NPC marker Nestin in neurospheres. The nuclei were stained with Hoechst 33342. c Immunofluorescent staining of OPC markers, including PDGFR $\alpha, N G 2$, and Olig2, in NPCderived OPCs. The nuclei were stained with Hoechst 33342. d Immunofluorescent staining of the OL marker MBP in OPCs induced to differentiate toward $\mathrm{OLs}$ in the presence of various concentrations of T3 for 4.5 days. e Dose-response of T3 in inducing $\mathrm{OPC}$ to OL differentiation. Data are mean $\pm \operatorname{SEM}(n=3)$. Scale bars, $100 \mu \mathrm{m}$

a single layer on poly-O-ornithine plus laminin-coated plates in OPC medium supplemented with bFGF and PDGF-AA. After 2 days, the majority of the cells were differentiated into OPCs, characterized by the expression of the platelet-derived growth factor receptor a (PDGFRa), the proteoglycan NG2, and the OL-specific transcription factor Olig2 (Fig. 1c). After the removal of the growth factors, OPCs differentiated toward OLs, and the marker of mature OLs, MBP, could be detected (Fig. 1d) after 4.5 days. T3, a wellknown inducer of OPC differentiation [21, 22], dose-dependently enhanced the generation of $\mathrm{MBP}^{+} \mathrm{OLs}$ (Fig. 1d, e).

Using this differentiation assay, we screened a small library that contained many old drugs and pathway regulators. NPC-derived OPCs were treated with vehicle $(0.2 \%$, v/v, DMSO), T3 $(100 \mathrm{nM}$, positive control), or various compounds $(20 \mu \mathrm{M})$ for 4.5 days, and the percent of $\mathrm{MBP}^{+}$cells was subsequently used to evaluate the efficacy of the compounds. Donepezil, an AChEl which is used to treat $A D$, was found to increase the percent of $\mathrm{MBP}^{+}$cells in the primary screen (Fig. 2a). To confirm the result and evaluate the best concentration of donepezil for OPC to OL differentiation, NPC-derived OPCs were treated with various concentrations $(5-40 \mu \mathrm{M})$ of donepezil in differentiation medium. Donepezil was found to promote OPC to OL differentiation, and the effect plateaued at $20 \mu \mathrm{M}$ (Fig. 2b, c). 

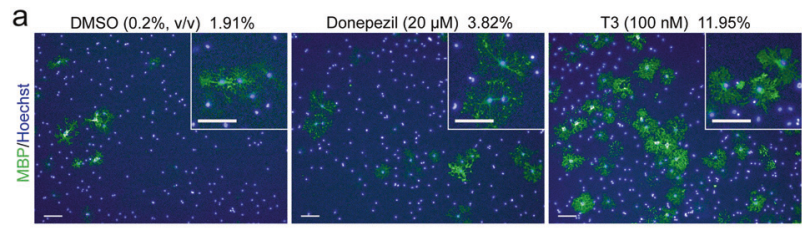

b
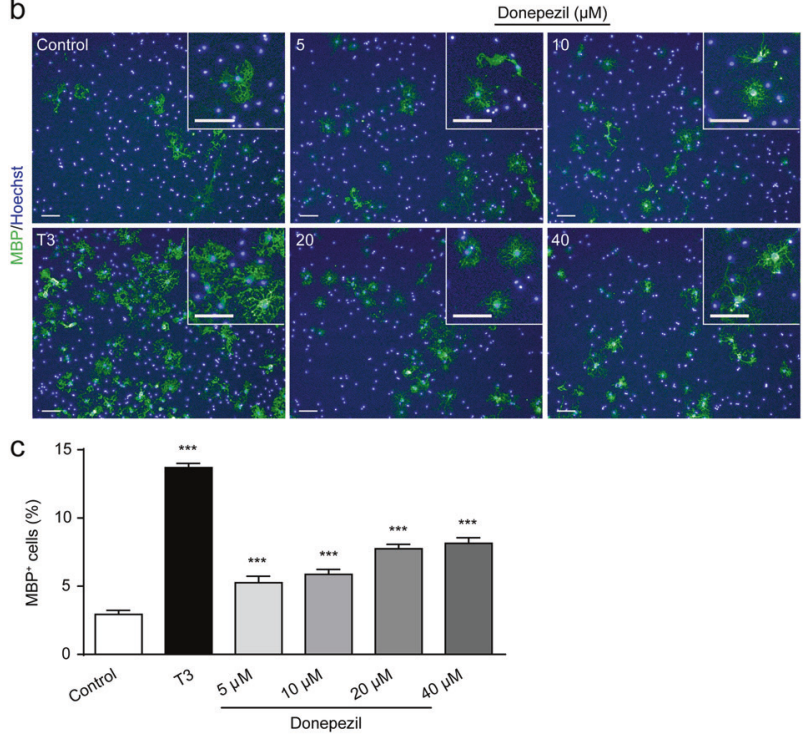

Fig. 2 Donepezil promotes OL differentiation from NPC-derived OPCs. a Representative images from the initial screening. NPCderived OPCs were induced to differentiate toward OLs in the presence of vehicle control (0.2\% DMSO), donepezil $(20 \mu \mathrm{M})$, and T3 $(100 \mathrm{nM})$ for 4.5 days. Cells were then immunostained for MBP (green) and Hoechst 33342 (blue). b NPC-derived OPCs were induced to differentiate in the presence of various concentrations of donepezil $(5-40 \mu \mathrm{M})$ for 4.5 days. Representative images of $\mathrm{MBP}^{+}$ cells. c Quantification of the percentage of $\mathrm{MBP}^{+}$cells presented in b. Data are the mean \pm SEM $(n=6),{ }^{* * *} P<0.001$ vs. control (Student's $t$-test). Scale bars, $100 \mu \mathrm{m}$

Donepezil, but not other AChEls, promotes OPC to OL differentiation

Donepezil is a selective AChEl that is widely used for the treatment of AD. We wondered whether other AChEls have similar effects on OPC differentiation. Three AChEls, including huperzine A, rivastigmine, and tacrine, were tested in the OPC differentiation system in doses that ranged from 5 to $40 \mu \mathrm{M}$. To our surprise, with the exception of donepezil, these AChEls did not increase the number of $\mathrm{MBP}^{+}$cells in all concentrations tested (Fig. 3a). Furthermore, qPCR analysis indicated only donepezil treatment induced an increase of $\mathrm{OL}$ lineage genes, including CNPase, MAG, MBP, and PLP, at the mRNA level (Fig. 3b). Moreover, the increased expression of MBP protein by the treatment of donepezil was further confirmed with Western blot analysis (Fig. $3 c_{1} d$ ). Collectively, donepezil, but not other AChEls, promotes OPC to OL differentiation, which suggests that donepezil's effect in promoting OPC differentiation is not dependent on the inhibition of AChE.

Donepezil enhances myelination in OPC-DRG neuron co-culture in vitro

Donepezil's effect in promoting OPC to OL differentiation was further assessed in primary OPCs. Mouse primary OPCs were cultured in differentiation medium supplemented with various concentrations of donepezil $(2.5-20 \mu \mathrm{M})$ for 4 days and then fixed and immunostained for MBP. The primary OPCs displayed a better differentiation potential than the NPC-derived OPCs without

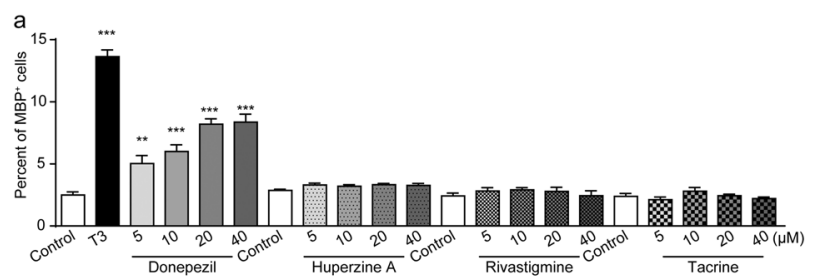

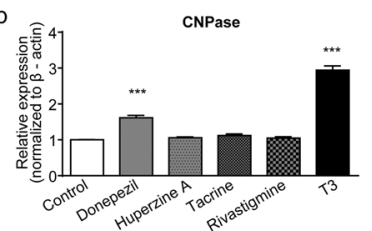
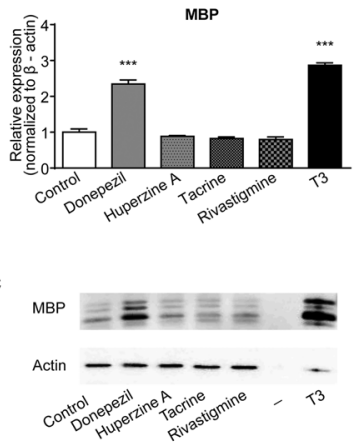

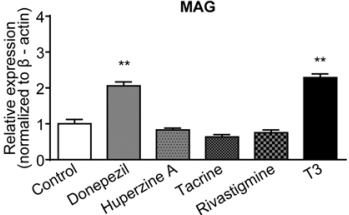

PLP

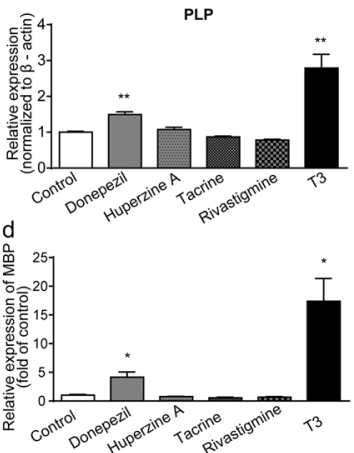

Fig. 3 Comparison between donepezil and other AChEls in inducing OPC differentiation and myelin-related gene expression. a Statistical analysis of $\mathrm{MBP}^{+}$cells generated from NPC-derived OPCs treated with various AChEls, including donepezil, huperzine A, rivastigmine, and tacrine, for 4.5 days. Data are representative of three independent experiments, mean \pm SEM $(n=6),{ }^{* *} P<0.01,{ }^{* * * *} P<$ 0.001 vs. control (Student's $t$-test). b Realtime q-PCR analysis of CNPase, myelin-associated glycoprotein (MAG), MBP, and proteolipid protein (PLP) expression in cells cultured with various AChEls (20 $\mu \mathrm{M})$. The results were normalized to $\beta$-actin expression in the same sample and then normalized to the control. Data are the mean \pm SEM $(n=3),{ }^{* * *} P<0.01,{ }^{* * *} P<0.001$ vs. control (Student's $t$-test). c Western blot analysis of MBP in NPC-derived OPCs treated with various AChEls $(20 \mu \mathrm{M})$ for 4.5 days. d The level of MBP in c was quantified and first normalized to GAPDH in the same sample and then normalized to the control. Data are the mean $\pm \operatorname{SEM}(n=3)$, ${ }^{*} P<0.1$ vs. control (Student's $t$-test)

treatment (Fig. 4a, b vs. Fig. 2b, c, vehicle control). However, donepezil could still significantly increase the percentage of $\mathrm{MBP}^{+}$-OLs generated from the primary OPCs (Fig. 4a, b). Since donepezil was so efficient in promoting the differentiation of primary OPCs, we wondered whether it was as effective in promoting myelin formation. To address this question, we set up an in vitro myelination system by co-culturing DRG neurons isolated from postnatal (P5-P10) mice with primary OPCs. The effect of donepezil in promoting myelination was evaluated after 9 days of co-culture. Myelinated axons are positive for both the $\mathrm{OL}$ marker MBP and the axon marker NF-200 (neurofilament-200 kDa). Donepezil was found to increase the length of myelinated axons in the co-culture, and the best effect was observed at $10 \mu \mathrm{M}$ (Fig. 4c, d).

Donepezil promotes remyelination in vivo

Cuprizone-induced demyelination in mice is a commonly used animal model to investigate the demyelination and remyelination processes $[23,24]$. We subsequently examined the efficacy of donepezil in enhancing remyelination in vivo in the cuprizoneinduced demyelination model. Adult female C57BL/6 mice were fed a diet that contained $0.2 \%(\mathrm{w} / \mathrm{w}$ ) cuprizone for 5 weeks, followed by 
a

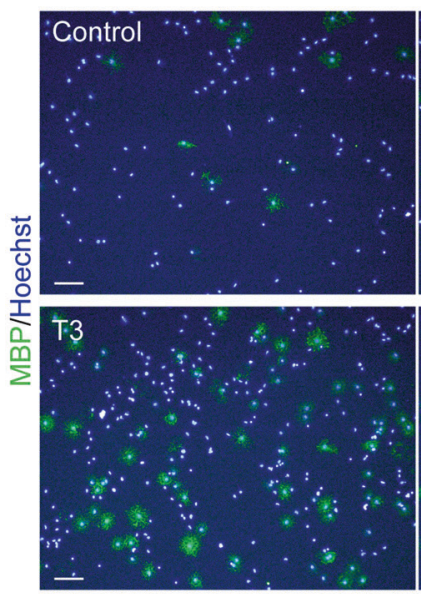

C

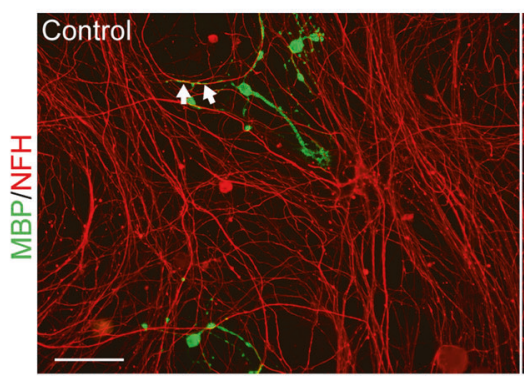

d

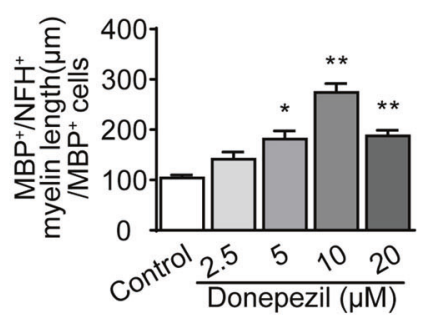

Donepezil $(\mu \mathrm{M})$
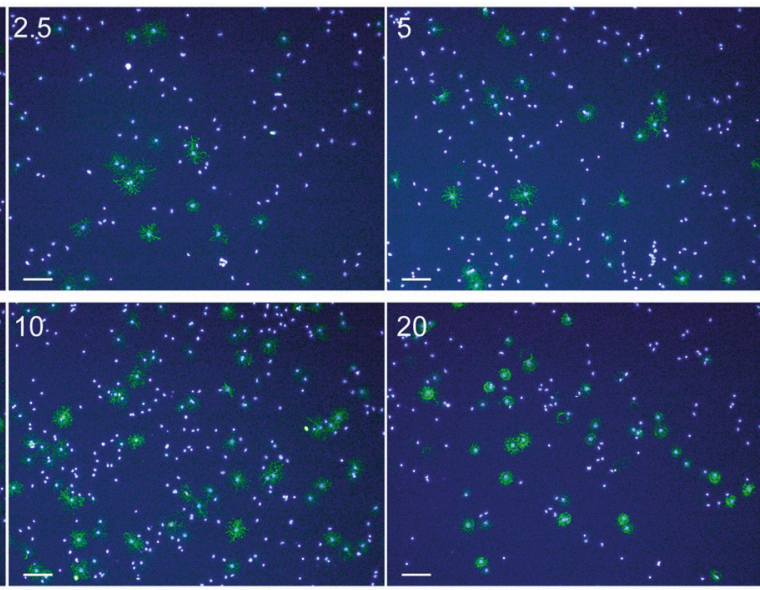

b

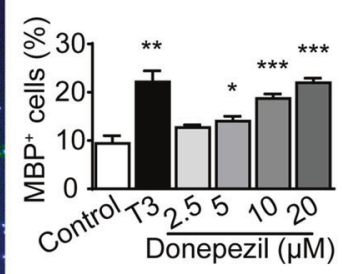

Donepezil $(\mu \mathrm{M})$
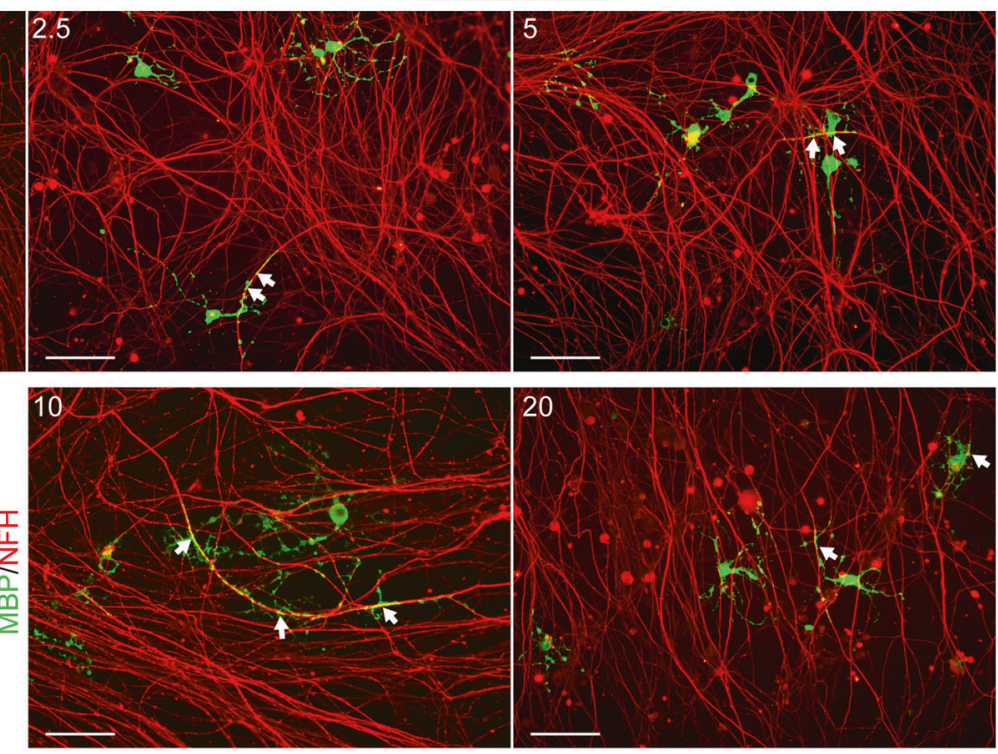

Fig. 4 Donepezil enhances primary OPC differentiation and in vitro myelination of co-cultured axons. a Representative images of $\mathrm{MBP}^{+}$cells from primary OPCs cultured with various concentrations of donepezil $(2.5-20 \mu \mathrm{M})$ for 4 days. Scale bars, $100 \mu \mathrm{m}$. b Quantification of the percentage of $\mathrm{MBP}^{+}$cells presented in a. Data are the mean \pm SEM $(n=6),{ }^{*} P<0.05,{ }^{* *} P<0.01{ }^{* * *} P<0.001$ vs. control (Student's $t$-test). c Primary OPCs were co-cultured with DRG-neurons and treated with vehicle or donepezil for 9 days. The cells were then fixed and stained for MBP (oligodendrocytes, green) and NF-200 (neurofilament, red). Arrows indicate myelinated axons (double positive for NF-200 and MBP). Scale bars, $100 \mu \mathrm{m}$. d Quantification of myelinated axons in OPC-DRG neuron co-cultures in the presence of various concentrations of donepezil for 9 days. Data are the mean \pm SEM $(n=3),{ }^{*} P<0.05,{ }^{* *} P<0.01$ vs. control (Student's $t$-test)

standard chow feeding with a daily intraperitoneal injection of donepezil (1.5 or $5 \mathrm{mg} / \mathrm{kg}$ ) or vehicle for $1-3$ weeks (Fig. 5a). The myelin status in the corpus callosum region was measured by Luxol fast blue staining. Five-week cuprizone treatment induced the complete loss of myelin in the corpus callosum as indicated by the loss of blue staining (Fig. 5b). After cuprizone removal, spontaneous myelin repair could be gradually observed in the vehicle group, and donepezil substantially accelerated the remyelination process, particularly after a 2-week treatment (Fig. 5b, c). We further investigated the degree of remyelination by immunofluorescent staining of the mature OL markers MBP and GST- $\pi$ in the corpus callosum after a 2-week treatment with donepezil. Donepezil significantly increased the MBP intensity and the number of GST$\pi^{+}$mature OLs in the corpus callosum compared with the vehicle treatment (Fig. $6 a-c)$. The Olig2 ${ }^{+}$OL-lineage cells were also significantly increased in the corpus callosum region of the mice treated with donepezil, while the PDGFRa ${ }^{+}$OPCs were reduced in the same region (Fig. $6 \mathrm{~d}-\mathrm{f}$ ).

The myelin status was further investigated with electron microscopy. The healthy naïve mice had a tightly wrapped myelin sheath. The cuprizone diet for 5 weeks led to severe demyelination in the corpus callosum (Fig. $6 \mathrm{~g}$ ). Two weeks after cuprizone withdrawal, the vehicle group showed significant spontaneous remyelination, and the donepezil-treated animals had more myelinated axons (Fig. $6 \mathrm{~g}, \mathrm{~h}$ ). The G-ratios (the ratio of the diameter of a given axon and the diameter of the axon plus myelin) of the remyelinated axons were evaluated. Donepezil not only increased the number of myelinated axons (Fig. 6h) but also reduced the G-ratio of the remyelinated axons (Fig. 6i), which indicates a better recovery. Taken together, donepezil promotes the generation of OLs and remyelination in the cuprizone-induced demyelination mouse model. 
a

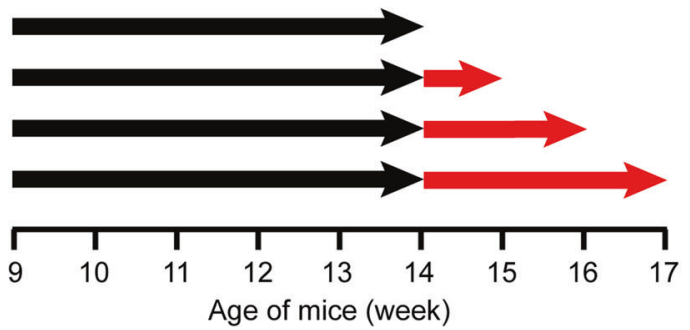

b
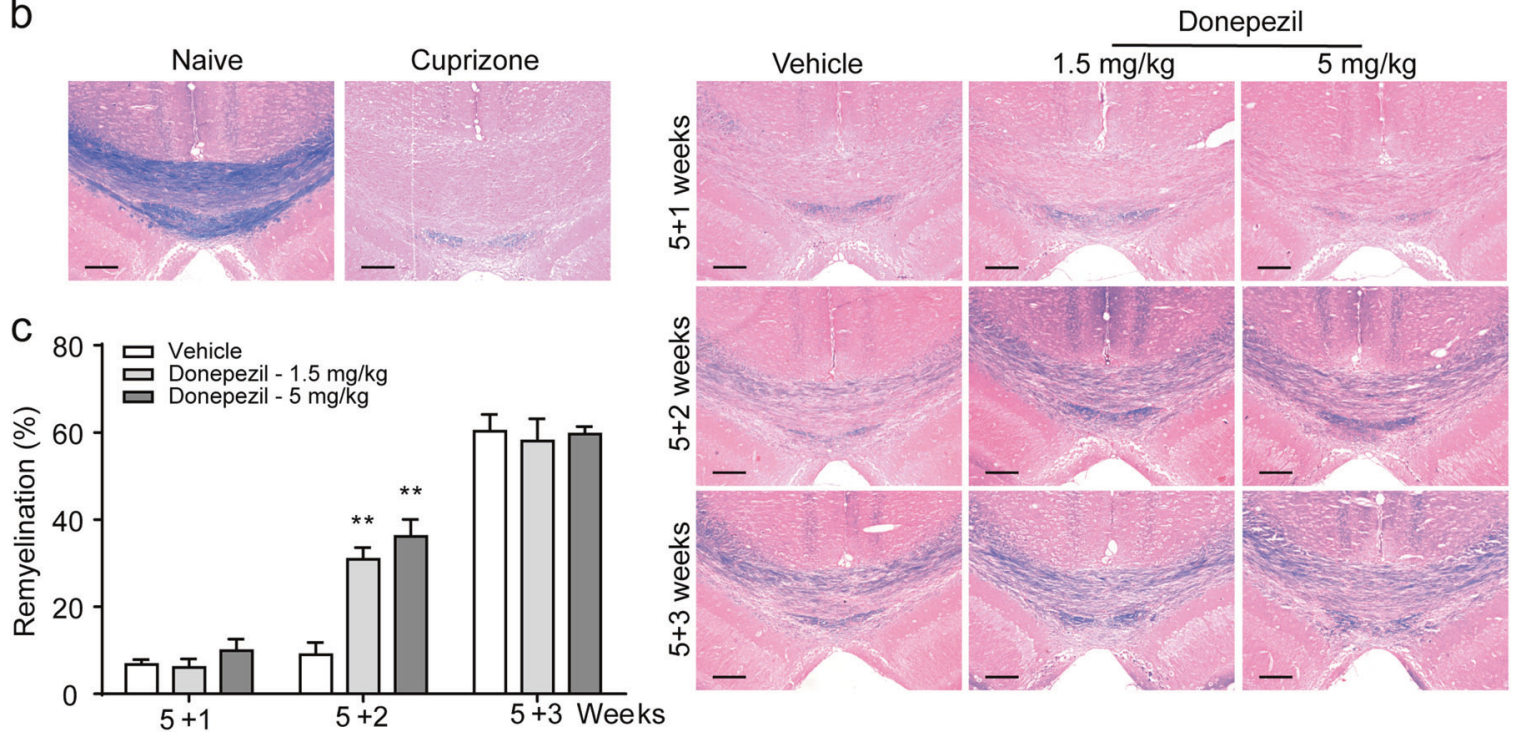

Fig. 5 Donepezil enhances remyelination in vivo in cuprizone-induced demyelination model. a A diagram of cuprizone-induced demyelination model and the administration of donepezil. Female adult C57BL/ 6 mice were fed a diet that contained $0.2 \%$ (w/w) cuprizone for 5 weeks, followed by a normal diet and donepezil (1.5 or $5 \mathrm{mg} / \mathrm{kg}$, intraperitoneal injection) treatment for 1, 2, or 3 weeks. b Representative images of the corpus callosum region stained with Luxol fast blue after cuprizone and donepezil treatment. Scale bars, $100 \mu \mathrm{m}$. c Statistical analysis of the myelinating areas in $\mathbf{b}$. Data are the mean \pm SEM (four mice per group; five sections from each mouse were analyzed); ${ }^{* *} P<0.01$ vs. vehicle (Student's $t$-test)

\section{DISCUSSION}

For a long time, donepezil has been used for the treatment of $A D$ with minimal side-effects because it is a reversible AChEl and enhances the ACh level in the brain. However, an increasing number of studies have suggested that donepezil has many other pharmacological properties. Takada and colleagues have demonstrated that donepezil protects rat cortical neurons against glutamate neurotoxicity via $a 4 \beta 2-$ and $a 7-n A C h R s$, but not $\mathrm{mAChRs}$ [17]. Donepezil also shows significant protection against A $\beta$-induced apoptosis in a neuroblastoma cell line [25]. In addition to its neuroprotective effects, donepezil has been demonstrated to exert anti-inflammatory actions. Donepezil could suppress TNFa-mediated intracellular $\mathrm{Ca}^{2+}$ elevation and NO production through the PI3K pathway in rodent microglia cells [26]. Donepezil has also been confirmed to alleviate the LPS-induced inflammatory response and suppress nuclear translocation of NF-KB in a murine macrophage cell line [20]. Recent studies have shown that donepezil could ameliorate clinical and pathological symptoms in EAE (experimental autoimmune encephalomyelitis) mice by reducing the permeability of the BBB (blood-brain barrier) [27]. In this study, donepezil is further demonstrated to promote $\mathrm{OL}$ generation from NPC-derived OPCs or primary OPCs isolated from the cerebral cortex, while other AChEls do not have the same effects. We also observed that donepezil treatment leads to a significant increase in myelin formation in OPC-DRG neuron coculture in vitro and the cuprizone-induced demyelination model in vivo.
The exact mechanism that underlies donepezil's effect in promoting OPC to OL differentiation remains unclear. Donepezil has been reported to directly interact with the $n A C h R s$ via complex mechanisms in an AChE-independent manner. Among the diverse nAChRs, $a 4 \beta 2-$ and $a 7-n A C h R s$ are the major types distributed in the CNS, while a7-nAChR is particularly located in the hippocampus and cerebral cortex $[28,29]$. Donepezil has been shown to upregulate nAChRs in cortex neurons [30]. Previous reports have also demonstrated that $\mathrm{nAChRs}$ are expressed on OPCs, but are not detectable after differentiation [31]. Moreover, the $\mathrm{nAChR}$ antagonist mecamylamine can partially inhibit the increase in the number of OLs following donepezil treatment [32]. These findings suggest that nAChRs might be involved in donepezil-promoted OL differentiation. In addition, donepezil has been found to protect against $A \beta$-induced neuronal cell death by activating the PI3K pathway [33]. Moreover, the PI3K/Akt pathway is also activated by myelinating factors, including neuregulins, integrins, and IGF-1 [34-37]. It has been shown that the expression of constitutively active AKT increases myelination in mice [38]. Therefore, the PI3K/Akt pathway might also be involved in donepezil-promoted OL differentiation.

In conclusion, this study demonstrates that donepezil can significantly promote OPC to OL differentiation and stimulate remyelination in a cuprizone-induced demyelination animal model. Considering the safe use of donepezil in neurodegenerative diseases, such as $A D$, for many years, it might be repurposed to treat demyelinating diseases, such as MS. 
a

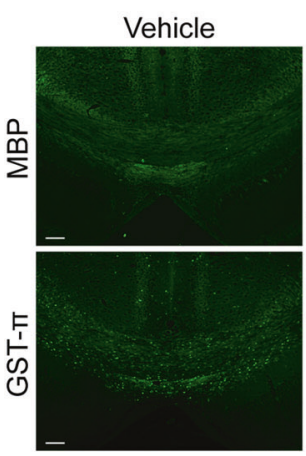

d
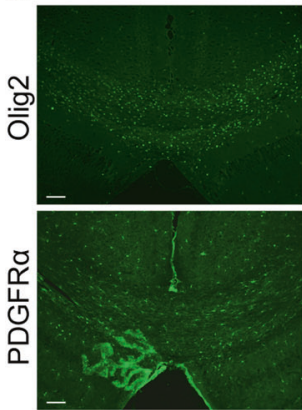

g

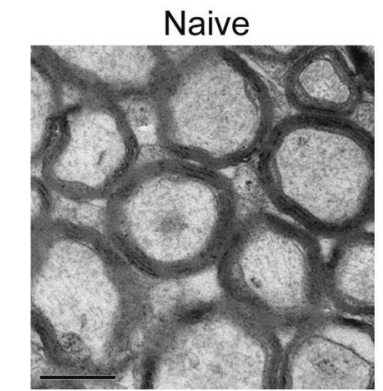

$\mathrm{h}$

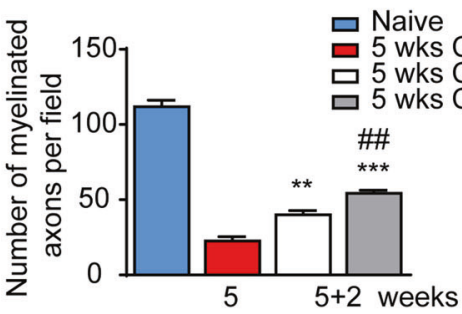

b

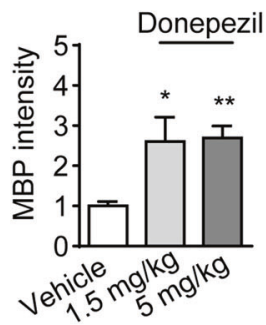

e
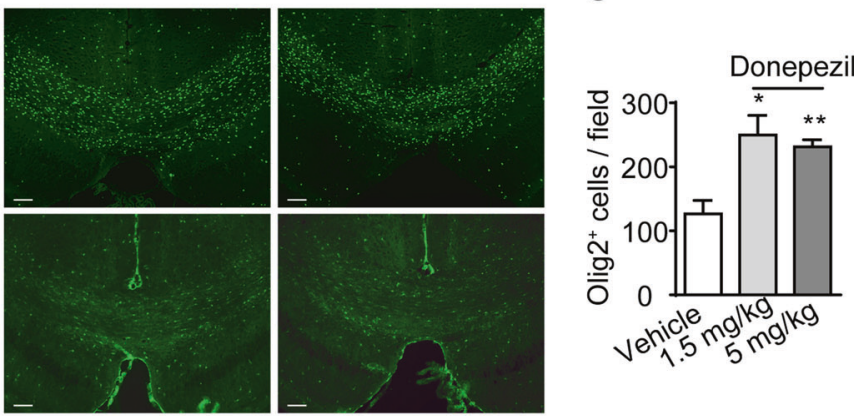

C

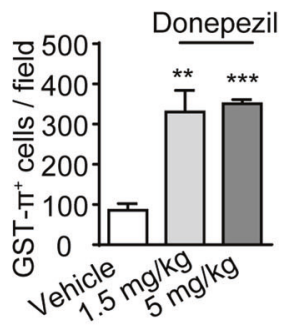

f

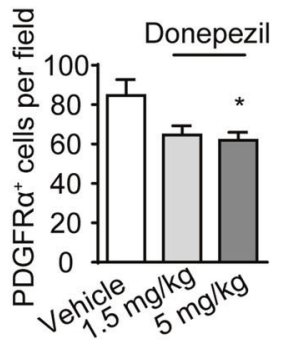

5 weeks

5 weeks cuprizone +2 weeks
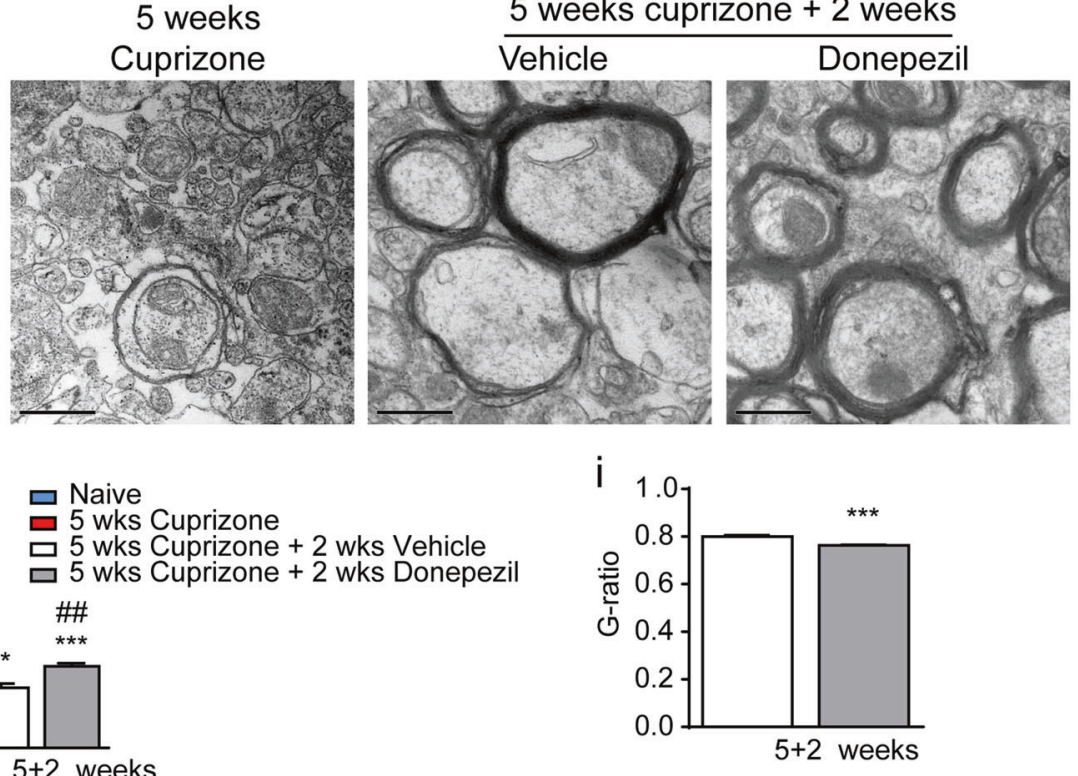

Fig. 6 Evaluation of remyelination in cuprizone model with fluorescence and electron microscopy. a Immunofluorescence staining of MBP and GST- $\pi$ in the corpus callosum isolated from cuprizone-fed mice treated with donepezil or vehicle for 2 weeks. Scale bars, $100 \mu \mathrm{m}$. b, c Statistical analysis of the MBP intensity and the number of GST- $\pi^{+}$cells presented in a. Data are the mean \pm SEM (four mice per group; five sections from each mouse were analyzed), ${ }^{*} P<0.05,{ }^{* *} P<0.01,{ }^{* * *} P<0.001$ vs. vehicle, Student's $t$-test. d Immunofluorescence staining of Olig 2 and PDGFR $\alpha$ in the corpus callosum isolated from cuprizone-fed mice treated with donepezil or vehicle for 2 weeks. Scale bars, $100 \mu \mathrm{m}$. e, $\mathbf{f}$ Statistical analysis of the number of Olig2 ${ }^{+}$and PDGFR $\alpha^{+}$cells presented in $\mathbf{d}$. Data are the mean \pm SEM (four mice per group; five sections from each mouse were analyzed), ${ }^{*} P<0.05,{ }^{* *} P<0.01$ vs. vehicle, Student's $t$-test. g Representative electron microscopy images of the corpus callosum region isolated from naïve mice, mice fed with cuprizone for 5 weeks, or cuprizone-fed mice treated with vehicle or donepezil (5 mg/ $\mathrm{kg}$ ) for an additional 2 weeks after cuprizone withdrawal. Scale bars, $0.2 \mu \mathrm{m}$. $\mathbf{h}$ Quantification of the myelinated axons from $\mathbf{g}$. Data are the mean \pm SEM (four mice per group; eight sections from each mouse were analyzed). ${ }^{* *} P<0.01,{ }^{* * *} P<0.001$ vs. cuprizone group, ${ }^{\# \#} P<0.01$ vs. vehicle group (one-way ANOVA followed by Tukey's multiple comparison test). i Quantification of the G-ratios of the remyelinated axons in g. Data are the mean \pm SEM ( $n=200 ; \sim 50$ axons counted per mouse; four mice per group), ${ }^{* * *} P<0.001$ vs. vehicle group (Student's $t$-test) 


\section{ACKNOWLEDGEMENTS}

This work was supported by grants from the Ministry of Science and Technology of China (No. 2015CB964503, 2014CB965002, and 2017YFA0104002), the Chinese Academy of Sciences (No. XDA16010202), and the National Natural Science Foundation of China (Nos. 81425024, 81472862, and 31501189).

\section{ADDITIONAL INFORMATION}

Competing interests: The authors declare no competing interests.

\section{REFERENCES}

1. Nave KA. Myelination and the trophic support of long axons. Nat Rev Neurosci. 2010;11:275-83.

2. Saab AS, Tzvetanova ID, Nave KA. The role of myelin and oligodendrocytes in axonal energy metabolism. Curr Opin Neurobiol. 2013;23:1065-72.

3. Levine JM, Reynolds R, Fawcett JW. The oligodendrocyte precursor cell in health and disease. Trends Neurosci. 2001;24:39-47.

4. Hughes EG, Kang SH, Fukaya M, Bergles DE. Oligodendrocyte progenitors balance growth with self-repulsion to achieve homeostasis in the adult brain. Nat Neurosci. 2013;16:668-76.

5. Young KM, Psachoulia K, Tripathi RB, Dunn SJ, Cossell L, Attwell D, et al. Oligodendrocyte dynamics in the healthy adult CNS: evidence for myelin remodeling. Neuron. 2013;77:873-85.

6. Wolswijk G. Oligodendrocyte survival, loss and birth in lesions of chronic-stage multiple sclerosis. Brain. 2000;123:105-15.

7. Liu Y, Zhou J. Oligodendrocytes in neurodegenerative diseases. Front Biol. 2013;8:127-33.

8. Baumann N, Pham-Dinh D. Biology of oligodendrocyte and myelin in the mammalian central nervous system. Physiol Rev. 2001;81:871-927.

9. Popescu BF, Lucchinetti CF. Pathology of demyelinating diseases. Annu Rev Pathol. 2012;7:185-217.

10. Deshmukh VA, Tardif V, Lyssiotis CA, Green CC, Kerman B, Kim HJ, et al. A regenerative approach to the treatment of multiple sclerosis. Nature. 2013;502:327-32.

11. Magalon K, Zimmer C, Cayre M, Khaldi J, Bourbon C, Robles I, et al. Olesoxime accelerates myelination and promotes repair in models of demyelination. Ann Neurol. 2012;71:213-26.

12. Magalon K, Le Grand M, El Waly B, Moulis M, Pruss R, Bordet T, et al. Olesoxime favors oligodendrocyte differentiation through a functional interplay between mitochondria and microtubules. Neuropharmacology. 2016;111:293-303.

13. Du C, Duan Y, Wei W, Cai Y, Chai H, Lv J, et al. Kappa opioid receptor activation alleviates experimental autoimmune encephalomyelitis and promotes oligodendrocyte-mediated remyelination. Nat Commun. 2016;7:11120.

14. Guo YE, Suo N, Cui X, Yuan Q, Xie X. Vitamin C promotes oligodendrocytes generation and remyelination. Glia. 2018;66:1302-16.

15. Shaikh S, Verma A, Siddiqui S, Ahmad S, Rizvi S, Shakil S, et al. Current acetylcholinesterase-inhibitors: a neuroinformatics perspective. CNS Neurol Disord Drug Targets. 2014;13:391-401.

16. Colovic MB, Krstic DZ, Lazarevic-Pasti TD, Bondzic AM, Vasic VM. Acetylcholinesterase inhibitors: pharmacology and toxicology. Curr Neuropharmacol. 2013;11:315-35.

17. Takada $Y$, Yonezawa A, Kume $T$, Katsuki $H$, Kaneko $S$, Sugimoto $H$, et al. Nicotinic acetylcholine receptor-mediated neuroprotection by donepezil against glutamate neurotoxicity in rat cortical neurons. J Pharmacol Exp Ther. 2003;306:772-7

18. Takada-Takatori Y, Kume T, Ohgi Y, Izumi Y, Niidome T, Fujii T, et al. Mechanism of neuroprotection by donepezil pretreatment in rat cortical neurons chronically treated with donepezil. J Neurosci Res. 2008;86:3575-83.
19. Solntseva El, Kapai NA, Popova OV, Rogozin PD, Skrebitsky VG. The involvement of sigma1 receptors in donepezil-induced rescue of hippocampal LTP impaired by beta-amyloid peptide. Brain Res Bull. 2014;106:56-61.

20. Arikawa M, Kakinuma Y, Noguchi T, Todaka H, Sato T. Donepezil, an acetylcholinesterase inhibitor, attenuates LPS-induced inflammatory response in murine macrophage cell line RAW 264.7 through inhibition of nuclear factor kappa B translocation. Eur J Pharmacol. 2016;789:17-26.

21. Barres BA, Lazar MA, Raff MC. A novel role for thyroid hormone, gluocorticoids and retinoic acid in timing oligodendrocyte development. Development. 1994;120:1097-108.

22. Billon $\mathrm{N}$, Tokumoto $\mathrm{Y}$, Forrest $\mathrm{D}$, Raff $\mathrm{M}$. Role of thyroid hormone receptors in timing oligodendrocyte differentiation. Dev Biol. 2001;235:110-20.

23. Kipp M, Clarner T, Dang J, Copray S, Beyer C. The cuprizone animal model: new insights into an old story. Acta Neuropathol. 2009;118:723-36.

24. Matsushima GK, Morell P. The neurotoxicant, cuprizone, as a model to study demyelination and remyelination in the central nervous system. Brain Pathol. 2001;11:107-16.

25. Arias E, Gallego-Sandin S, Villarroya M, Garcia AG, Lopez MG. Unequal neuroprotection afforded by the acetylcholinesterase inhibitors galantamine, donepezil, and rivastigmine in SH-SY5Y neuroblastoma cells: Role of nicotinic receptors. J Pharmacol Exp Ther. 2005;315:1346-53.

26. Haraguchi Y, Mizoguchi Y, Ohgidani M, Imamura Y, Murakawa-Hirachi T, Nabeta $\mathrm{H}$, et al. Donepezil suppresses intracellular $\mathrm{Ca}^{2+}$ mobilization through the PI3K pathway in rodent microglia. J Neuroinflammation. 2017;14:258.

27. Jiang Y, Zou Y, Chen S, Zhu C, Wu A, Liu Y, et al. The anti-inflammatory effect of donepezil on experimental autoimmune encephalomyelitis in C57 BL/6 mice. Neuropharmacology. 2013;73:415-24.

28. Wada E, Wada K, Boulter J, Deneris E, Heinemann S, Patrick J, et al. Distribution of alpha 2, alpha 3, alpha 4, and beta 2 neuronal nicotinic receptor subunit mRNAs in the central nervous system: a hybridization histochemical study in the rat. J Comp Neurol. 1989;284:314-35.

29. Seguela P, Wadiche J, Dineley-Miller K, Dani JA, Patrick JW. Molecular cloning, functional properties, and distribution of rat brain alpha 7: a nicotinic cation channel highly permeable to calcium. J Neurosci. 1993;13:596-604.

30. Kume T, Sugimoto M, Takada $Y$, Yamaguchi T, Yonezawa A, Katsuki H, et al. Upregulation of nicotinic acetylcholine receptors by central-type acetylcholinesterase inhibitors in rat cortical neurons. Eur J Pharmacol. 2005;527:77-85.

31. Sharma G, Vijayaraghavan S. Nicotinic receptor signaling in nonexcitable cells. J Neurobiol. 2002;53:524-34.

32. Imamura $O$, Arai $M$, Dateki $M$, Ogata $T$, Uchida $R$, Tomoda $H$, et al. Nicotinic acetylcholine receptors mediate donepezil-induced oligodendrocyte differentiation. J Neurochem. 2015;135:1086-98.

33. Noh MY, Koh SH, Kim Y, Kim HY, Cho GW, Kim SH. Neuroprotective effects of donepezil through inhibition of GSK-3 activity in amyloid-beta-induced neuronal cell death. J Neurochem. 2009;108:1116-25.

34. Taveggia C, Zanazzi G, Petrylak A, Yano H, Rosenbluth J, Einheber S, et al. Neuregulin-1 type III determines the ensheathment fate of axons. Neuron. 2005;47:681-94.

35. Barros CS, Nguyen T, Spencer KS, Nishiyama A, Colognato H, Muller U. Beta1 integrins are required for normal CNS myelination and promote AKT-dependent myelin outgrowth. Development. 2009;136:2717-24.

36. Carson MJ, Behringer RR, Brinster RL, McMorris FA. Insulin-like growth factor I increases brain growth and central nervous system myelination in transgenic mice. Neuron. 1993;10:729-40.

37. Ness JK, Mitchell NE, Wood TL. IGF-I and NT-3 signaling pathways in developing oligodendrocytes: differential regulation and activation of receptors and the downstream effector Akt. Dev Neurosci. 2002;24:437-45.

38. Flores Al, Narayanan SP, Morse EN, Shick HE, Yin X, Kidd G, et al. Constitutively active Akt induces enhanced myelination in the CNS. J Neurosci. 2008;28: 7174-83. 